\title{
Communications concerns for reused electric vehicle batteries in smart grids.
}

\author{
L. Canals Casals, B. Amante García
}

\begin{abstract}
Electric vehicles use a 10 to $25 \mathrm{kWh}$ battery. After its use in cars, these batteries are still in good conditions to be used for energy storage in stationary applications and in smart grid systems at all scales: Generation, transmission and distribution. However, before their reuse, there are some transformations to be done on these batteries, such as the ones concerning the communications. The electric vehicle batteries use a Battery Management System that controls the functionality and safety, transmitting its conditions and status but it also contains confidential information. This paper will study two strategies to deal with communications difficulties on their second life as storage energy devices.
\end{abstract}

Index Terms - Electric Vehicle, Batteries, Re-use, second life, Battery Management System.

\section{INTRODUCTION}

C URRENT electric vehicles (EV) are mostly powered by lithium batteries, which lose performance properties and get degraded by time and use. In order to ensure driving comfort and safety, it is considered that a battery is not useful for traction purposes when it has lost a $20 \%$ of its initial capacity [1], which is expected to happen after 8 to 10 years or after running more than $100.000 \mathrm{~km}$. But even in then, these batteries are quite performant for other applications such as renewable energy support [2], load balance and other stationary applications and services to the grid. This is called the second life or re-use of EV batteries.

Although possible and environmentally profitable, these reused batteries should compete with other battery technologies (such as common Lead acid batteries, Flow batteries or Nickel based batteries among others) in terms of performances, lifespan and, mostly, price.

Technically, there are different strategies to re-use EV batteries, going from the battery dismantle and combining modules to build a new one, to the direct re-use, which has not much manipulation apart from testing. Clearly, it is the direct re-use strategy the one having fewer costs [3]. In addition, this strategy is more accepted by car manufacturers as their product is less manipulated having fewer risks of failure.
Finally, space and weight are not that relevant in stationary applications as they are for traction purposes, so the extra costs of dismantling and re-building a battery are not expected to be compensated in most of the stationary cases. Thus, his study analyzes the communications needs and possible adaptations of direct re-used batteries.

These Li-ion batteries need control and supervision parameters. It is dangerous to overcharge or under-discharge them; The C-rates and working performances under the safety temperature windows are limited and, sometimes, voltage range is extra limited in order to ensure longer life cycle.

This control and restriction activities are handled by the Battery Management System (BMS) or the Battery Manage Control (BMC) [4]. In the case of EV batteries, the BMS is placed inside the battery pack, which is completely sealed, and should not be removed or manipulated. The BMS has also specific, precise and confidential algorithms, such as the state of health (SOH) or the state of charge (SOC) algorithms among others and security parameters that car manufacturers do not want to share neither give the possibility to modify them.

To understand how these BMS are prepared, an analysis of the EV communication system is presented. In the car, the EV batteries share and receive information with the onboard charger (OBC), the electric machine, the engine control units, and other car components. This information exchange is done using the Controlled Area Network (CAN) protocol defined especially for vehicles (ISO 11898). The information goes along the CAN bus (a two plait wire) in a sequence of square voltage signals or strings.

Having the origin on EVs, the $2^{\text {nd }}$ life could be understood as the step beyond the vehicle to grid (V2G) concept. The V2G interconnection use different standards depending on the world region, the objective pursued (which may go from intelligent charging to grid energy services [5]) and car manufacturer. As an example, the ISO 15118 using Power line Communication (PLC) system is used in Europe whereas Chademo is most common in Japan. However, in all these cases, it is the car OBC, not the BMS, the element connected to the Electric Vehicle Supply Equipment (EVSE), which is connected to the grid elements or utilities using other standards such as IEC 61850, OCPP, OICP, etc [6]. Thus, as the $2^{\text {nd }}$ life of EV batteries will use no OBC, neither an EVSE to manage the storage system, no advantage can be taken from V2G solutions and another approach should be studied based 
mainly on the battery.

EV batteries are all but standardized, each car manufacturer uses his own technology and package, the battery form is different for each car and model and so it is the refrigeration system [7]. They are built by the addition in series of various cells forming modules of certain voltage and capacity and then grouping several modules into battery packs (Fig. 1). The number of cells forming a battery depends of the type of cells, the power and the capacity of the battery that each manufacturer defines for the car model. E.g. the Renault Twitzy has 42 cells, the e-golf has 264 cells or the Tesla Model S has 7.104 cells.

Each of these cells has a cell-controller observing the voltage evolution, information that is send to the BMS. Additionally, each module has also a voltage evaluation and has, at least, one temperature sensor. Again, the BMS gathers this information. The whole package has a refrigeration system, controlling the temperature the coolant (air or water), the fan revolutions, the pump regulation... Depending on the car manufacturer, vehicles have other elements controlling the battery: current meters, power calculations, $\mathrm{SOH}$ algorithms and prognosis, fuses, isolation checks, and many other security devices ensuring no danger while driving an EV.

After all, each EV battery has, easily, more than 500 signals and messages going from and to the battery and each car manufacturers codifies them differently.

Based in the experience learned from the Sunbatt project, which re-uses 4 PHEV batteries for stationary applications using solar panels, EV chargers and the grid [8], this study explores two alternatives to solve the particular communication's complexity of EV batteries' re-use and presents the minimum number of parameters that must be exchanged.

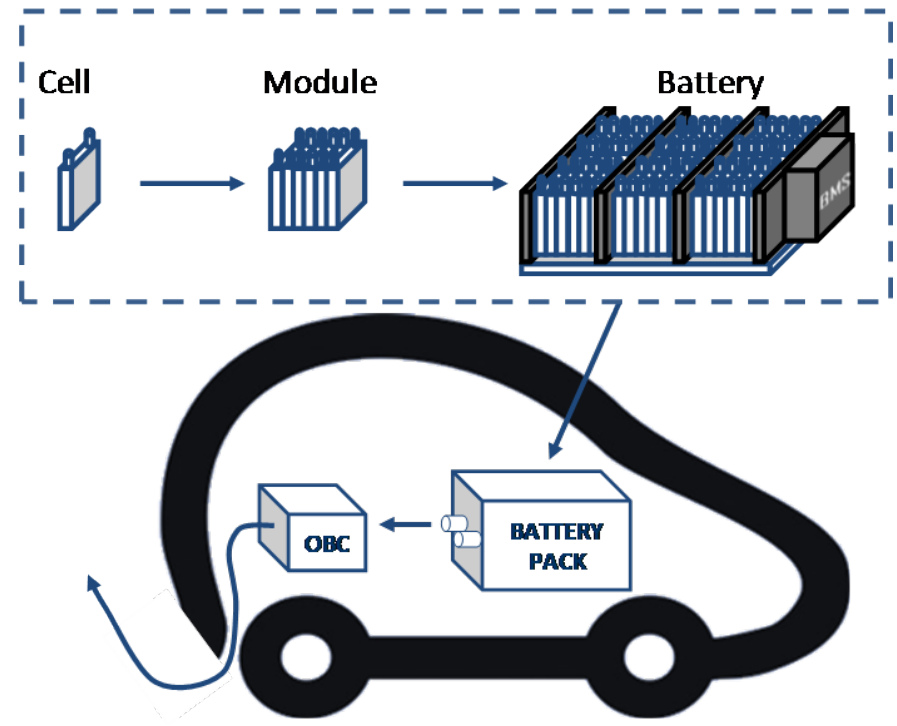

Fig. 1: EV battery composition from cell to battery pack.

\section{APROACH}

In the first place, a description of the possible $2^{\text {nd }}$ life applications and the elements involved will be presented to further continue with the physical communication system and the possible protocols to be used in these fields and how they may match with the re-used battery configuration.

\section{A. Stationary applications batteries' environment}

Batteries may participate in all the stages of the energy grid, from the energy generation to the distributed energy resources and into home applications providing different services and benefits. The renewable energy generation is variable depending on weather conditions and yearly seasons; energy storage systems offer the opportunity to eliminate this disadvantage, ensuring the programmed generation and enhancing the integration of clean energy generators. Batteries can be used to balance the energy production and demand, being able to use the energy plants at their optimal level and reduce emissions or shut down polluting power plants. Additionally, they facilitate the decentralized generation serving as energy buffers and helping the energy area regulation. At a distribution scale, they can be used to improve the energy quality or to enlarge the installations' lifetime. Finally at home/building level, they offer the possibility to reduce the electricity bill by buying the energy at lower fares or by reducing the power factor tariff using the battery for peak shaving. This wide range of possibilities means that batteries may interact with all the elements composing a grid: from a wind turbine to a dishwashing machine.

Nevertheless, this does not implies that batteries should communicate with all the elements and grid participants at the same time. A wiser approach indicates that batteries are used as a support or slave element in the system that takes no energy management decisions, which are held by the Energy Management System (EMS). Thus, batteries just need to communicate their status and conditions to one or two elements at most, as presented in Fig. 2.

These are:

\section{a)}

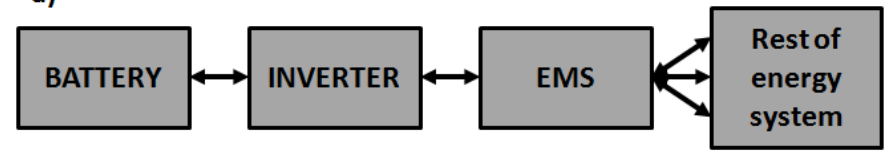

b)

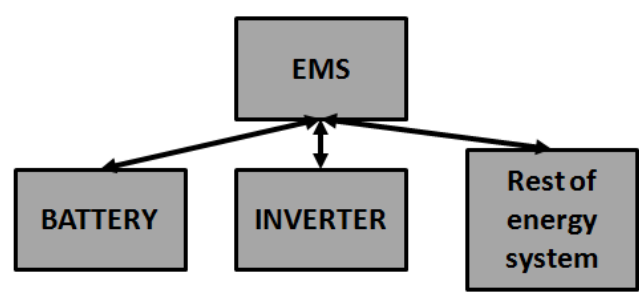

Fig. 2: Communications diagrams of the battery and the rest of elements in a grid. 
--The inverter that comes just after the battery so the energy will be transformed from DC to AC and vice-versa. It is the solution stablished in the Sunbatt project, where the 4 PHEV batteries are connected to the inverters.

--The energy management systems (EMS) that controls the loads, limitations and generation of the grid.

\section{B. Physical communications}

There are mainly three ways to communicate between electronic elements: The Shared Line systems, such as normal power or telephone lines; the specific lines, as IEEE1394, Ethernet, USB, etc.; and the wireless systems, such as Bluetooth, ZigBee, Z-wave, Wifi, Wimax or GSm among others. This section will propose the most suitable option considering the elements with whom the re-used EV battery has to communicate.

In the first case analyzed (Fig. 2 a), the battery is connected to the inverter and, this one, to the EMS, who takes the decisions. That is, the inverter works, in the communications field, as a transmitter from battery to EMS. Normally, the inverters use RJ-45/Ethernet/TCP-IP, RS-232 or two intertwined wires for CAN. Concurrently, as mentioned in the introduction, EV batteries exchange information via a CANbus using a specific codification of the CAN protocol based on the ISO 11898.

Hence, the most effective, economic and practical system to use for this first case (Fig. 2 a) is the communication via CAN, as both elements have it already implemented. The difficulties might come from the communication protocol and codification each one uses, which is going to be discussed in section II.C.

In the second case (Fig. 2 b), the battery is directly connected to the EMS. The EMS can be implemented in many electronic supports, the most used in automation are SCADA or other computer based systems, whereas HEMS is more specific for home applications. None of them use CANbus, they normally use TCP/IP or wireless connections.

In this second case, there is clearly a mismatch to be saved as the physical systems used are different. This can be solved by the use of bridge communication devices available in the market as a primary or temporarily solution. From an installer point of view, the battery re-use would be enhanced if EV batteries were prepared for the second life with an Ethernet or TCP/IP input/output connector. Howbeit, it is hard to foresee this option, as that would signify an extra cost for car manufacturers during the EV lifecycle that may not be used afterwards. In addition, car manufacturers might be able to sell these bridges as an extra service or a complement at the end of vehicles life.

Thus, the proposed system is similar to the configuration described in the work from Zhu et al. [9], where the management system uses Ethernet and the storage and load systems use CANbus for the fast and practical signals.

\section{Protocols}

There are multiple choices in the market of conventional batteries and BMS to select the ones that best suits the application's needs. However, in the introduction it was stated that re-used batteries would be delivered together with its BMS integrated, having no possibility to choose.

Although the BMS and most inverters use the CAN protocol, its configuration and signal codification is different. Therefore, a CAN matrix is absolutely necessary to decode the messages sent to and through the battery. However, car manufacturers are not willing to provide this CAN matrix, because that would mean direct access to the internal messages, the battery confidential information, and an undesired risk for parameters manipulations.

A possible solution for this incompatibility is the addition of a gateway that would allow the exchange of some of the messages but will block the others. In order to provide a higher security level, the information will be translated into different arrays, so the final receiver couldn't know the original CAN codification and protocol from the vehicle.

This Gateway should be understood not only as a firewall and translator but as an active element too. As mentioned in the introduction, the re-used battery expects some messages from the $\mathrm{OBC}$ in the car, as in stationary application there is no OBC nor a car, this gateway should provide these expected security messages to the BMS, such as "key position". For example, an industrial PC was used for this purpose in the Sunbatt project [10].

In the first case of study (Fig. 2 a), the inverter is expected to use the physical two braid wire connection from the battery. In order to have a public and open source protocol and codification, the selected communication protocol used by the gateway is proposed to be CANopen. This protocol was designed for automation applications and follows the CiA301 (CAN in automation) which is based, among others, on the same ISO 11898 used in the automotive industry. The bridges to pass from CAN to CANopen are easy to build, as CANopen is a higher level protocol, meaning that it adds terms in the data string identifying the application, the element sending the message and other significant characteristics and it contains in the end, the aspects like datalink and physical strings from the CAN protocol.

In the second case (Fig. 2 b), where the BMS is connected to the EMS, two options may be chosen: Either use a commercial bridge from CANopen to the protocol used in each case by the EMS (according to the physical system that best suits the specific installation (PLC, Wireless, Ethernet...)) or that the gateway provided by the car manufacturer, instead of giving the signal in CANopen, it provides it in Modbus or any other Ethernet compatible protocol (Fig. 3). This second option is less provable as there is a huge diversity of EMS programming. In some cases the EMS manufacturer is also providing solar panels, inverters and other smart grid elements using its own protocol to ensuring no communication failure. Consequently, some bridges would be needed anyway. 


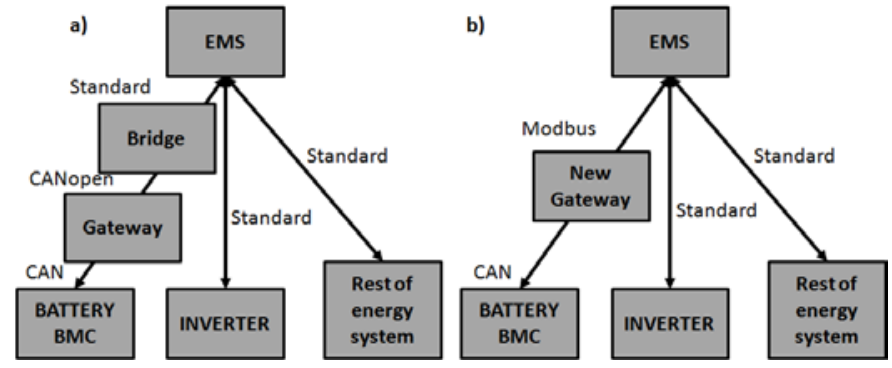

Fig. 3: Schema of the elements and the protocol used connected to the EMS.

As the communications between the inverter or EMS and the other devices in smart grids are already implemented in many applications, the protocol and physical transmission system presented in Fig. 3 are written as Standard.

After this analysis, it is found that, in the end, the solution that would fit in all cases is to use a gateway with CANopen protocol and, if needed, acquire a commercial bridge with the protocol and physical layer expected by the receiver or EMS.

\section{EXCHANGED INFORMATION WITH THE SMART GRID}

Taking the example of the Sunbatt project, this section analyzes the messages that should be selected and exchanged from the battery to the EMS or inverter. The Sunbatt project conceives the energy storage as a portable device that may be connected anywhere. That is the reason why the batteries, the cooling and refrigeration systems and the power electronic equipment are placed inside a maritime container as schematically presented in Fig. 4. In the particular case analyzed, the container is connected to $4 \mathrm{EV}$ chargers, solar panels and the local grid. The EMS is the element taking the energy management decision while the SCADA is the system that activates the different electronic elements. In future prototypes, it is expected that the EMS will be integrated in the SCADA. In the meanwhile, the SCADA concentrates and sends the information of all the elements to the EMS, working as a bridge. As mentioned in section II.C, the element described as PC in Fig. 4 should be understood as the gateway described all along the study. It connects the batteries' BMS with the inverters that are connected to the SCADA and EMS in the end. Additionally, although it could be executed by the EMS or SCADA, this PC is also connected to the cooling system of the container and refrigeration system of the batteries, which was adapted for space optimization.

Being a demonstrator, the Sunbatt container has also a Human Machine Interface (HMI) or screen to show up the functionalities and results. Finally, it has a small uninterrupted power supply (SAI in Fig. 4) to ensure the container shutdown.

The EMS is programmed following two strategies: to maximize economic profit and to reduce the $\mathrm{CO}_{2}$ emissions with $90 \mathrm{~kW}$ power limitation. To do so, the EMS receives information from outside the container: Grid pricings, in order to buy energy at lower fares and sell it (if permitted by local regulations) when it is expensive; Grid electricity

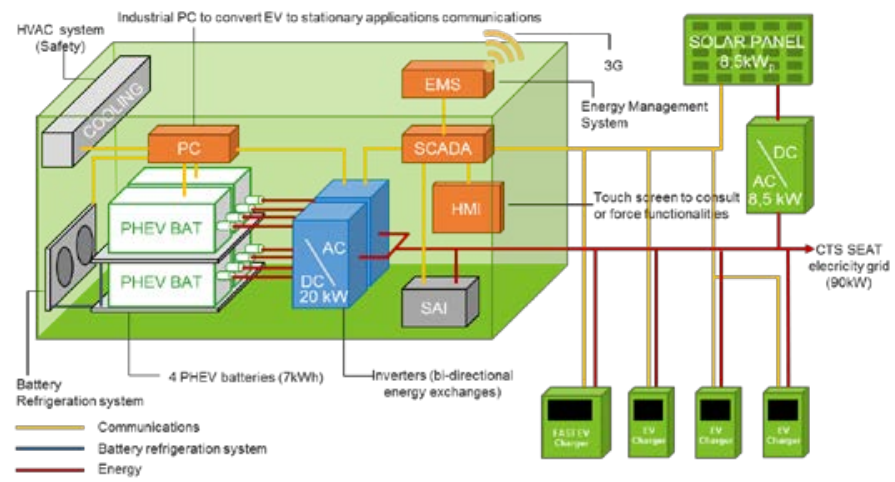

Fig. 4: Schema of the Sunbatt container connected to 3 normal EV chargers, one Fast EV charger, solar panels and a local grid.

generation's Mix to control the emissions; Meteorological forecast, so it can predict the Solar panels production and grid necessities in case of power supply emergencies and grid load predictions.

In this example, the batteries will absorb the extra energy produced by the solar panels if there is not enough consumption from the charging EVs and offer it back when there are sudden power interruptions, at night or to provide the necessary power at peak power demands when all chargers are simultaneously active. At the same time, the batteries are able to store energy when the cost of electricity or the electricity Mix is low to use it when the fares are higher or the energy generation is polluting.

In order to manage the energy properly, a part from load and demand prognosis, the EMS needs some information of the state of the battery. In this section a proposal of the minimum data needed for a correct energy management using $2^{\text {nd }}$ life batteries will be reasoned. See the summary in table I.

The battery power depends on its voltage (V) and current (I). Although these parameters can be evaluated from outside the battery, for security issues it is useful to validate that the voltage and intensity through the battery and inverter coincide. In order to know the maximum power the battery can offer, the EMS should know the maximal current $\left(I_{\max }\right)$ the battery can deliver at every instant.

This $\mathrm{I}_{\max }$ depends on SOC and temperature (T) of the battery. Its limitations are controlled by the BMS inside the battery. Additionally, the EMS should be able to provide power along time and not incur into sudden and unexpected current limitations. Consequently, it should know the maximal sustained current ( $\mathrm{I}_{\text {max_cont }}$ ). Similarly to current, voltage is also limited. The voltage in a battery varies depending on SOC, I, T and other chemical mechanisms [11], being its limits controlled by the BMS. Thus, the EMS might need this information for maximal and minimal Power $\left(\mathrm{P}_{\max }\right.$ and $\left.\mathrm{P}_{\min }\right)$ calculations.

As it has just been specified, SOC and $\mathrm{T}$ do have an impact on the battery performance. Temperature could be used, for example, to activate the refrigeration system that warrants the battery durability. Thus, it is necessary to receive this signal and the temperature working limits $\left(\mathrm{T}_{\max }, \mathrm{T}_{\min }\right)$.

SOC provides information of the quantity of energy still 
TABLE I:

SIGNALS EXCHANGE BETWEEN THE GATEWAY AND THE EMS.

\begin{tabular}{cclcc}
\hline \hline Parameter (unit) & Name & \multicolumn{1}{c}{ Use } & Type & From \\
\hline Voltage (V) & $\mathrm{V}$ & For instant power calculations. & Variable & Battery \\
Maximum Voltage (V) & $\mathrm{V}_{\max }$ & Future charge power calculations & Constant & Battery \\
Minimum Voltage (V) & $\mathrm{V}_{\min }$ & Future discharge power calculations & Constant & Battery \\
Current (A) & $\mathrm{I}$ & For security issues. & Variable & Battery \\
Max. Current & $\mathrm{I}_{\max }$ & For Power and energy calculations & Variable & Battery \\
Continuous Max. Current & $\mathrm{I}_{\max \text { cont }}$ & For Power and energy calculations & Variable & Battery \\
Temperature (K) & $\mathrm{T}$ & For battery working conditions optimization & Variable & Battery \\
Maximum Temperature (K) & $\mathrm{T}_{\max }$ & For security issues. & Constant & Battery \\
Minimum Temperature (K) & $\mathrm{T}_{\min }$ & For security issues. & Constant & Battery \\
Initial Capacity (Ah) & Capi & For energy calculations. & Constant & Battery \\
State of Charge & $\mathrm{SOC}$ & For energy calculations. & Variable & Battery \\
State of Health & $\mathrm{SOH}$ & For energy calculations and for maintenance planning. & Variable & Battery \\
Battery Identifier & $\mathrm{Id}$ & To manage correctly the energy distribution. & Constant & Gateway \\
Battery working mode & Mode & To allow balancing and maintenances. & Variable & Battery \\
\hline \hline
\end{tabular}

available in the battery. Conceptually, it is defined as the ratio between the energy in the battery and its total capacity.

However, batteries become less and less performing along time and use. This phenomenon is called capacity loss or aging [12], which is evaluated by $\mathrm{SOH}$ and it is defined by the division between the actual capacity by the initial capacity $\left(\mathrm{Cap}_{\mathrm{i}}\right)$. The capacity is measured in Ah.

Then, it can be seen that only three of these parameters are necessary. As the BMS controls the SOC and has the information of its $\mathrm{Cap}_{i}$, these two parameters are selected. The other necessary parameter is, which $\mathrm{SOH}$ may be estimated by different methods [12]-[14]. In fact, each car manufacturer uses its own designed algorithms to do so. This algorithms are part of the confidential information that should not be exposed elsewhere.

The designed storage system might have one or multiple batteries connected to the EMS [15]. Having more than one battery connected simultaneously is a situation not foreseen during the EV battery's design, as it works alone in the vehicle. For this reason, in almost all cases the re-used batteries do not have an identifier. This identification number (Id) should be added by the gateway so the EMS can activate the desired battery.

The last signal to be exchanged by these elements is the working mode of the battery. In EV this modes switches between Off, Stand-by, Charging, Driving and Balancing. The normal status of a battery in use in stationary applications will be "drive", as it is the only status that allows both discharging and charging (through regenerative braking) situations. However, EV batteries consist of many cells connected in series and parallel, and the cells perform softly different. Hence, it is needed to perform a cell battery balancing periodically. The best way to balance the cells in a battery is under no solicitation, i.e. the battery does it when there is no energy requirement, when the voltage is stabilized and without any need of external connection. It is the BMS defined by the car manufacturer who'll be in charge of the cell balance.

To sum up, all the needed parameters and their use are presented in Table II. Although this section is based in the Sunbatt project, the same parameters could be used for any other purpose.

From all the messages the BMS manages, which are more than 500, most of them are useless for the EMS on stationary applications. Indeed just the 14 messages from Table I are necessary.

\section{CONCLUSIONS}

Nowadays, a gateway is needed to connect EV re-used batteries to an inverter or EMS. In both cases the battery connection will be done by a two braid wire to the gateway. It is proposed that this gateway should also have a two braid wire using the CANopen protocol when connected to the inverter. On the contrary, if it is connected to the EMS, the gateway output should be one of the accepted by EMS or another commercial bridge should be added.

The proposed gateway incorporates a CAN matrix that changes the Battery CAN codification to CANopen, protecting the internal battery parameters and its algorithms. Additionally, this gateway should provide the responses expected by the battery to simulate the car conditions.

From all the messages (more than 500) exchanged by the battery and the rest of the EV, only 14 messages are needed by the EMS.

If this proposal is taken into consideration by car and battery manufacturers, it would facilitate the implementation of storage devices into smart and micro grids. The signals proposed to be exchanged with the EMS have no confidentiality impediments and the CAN matrix installed in the gateway could be sold by the same car manufacturers, ensuring no intromission and expanding the after-sales business.

\section{ACKNOWLEDGEMENTS}

The authors want to thank the UPC and SEAT for the opportunity to investigate in the second life of EV batteries.

Moreover, the authors thank the Sunbatt partners for their implication in the project and specially ACCIÓ for funding it. 


\section{REFERENCES}

[1]

B. E. Olivares, M. a. Cerda Munoz, M. E. Orchard, and J. F. Silva, "Particle-Filtering-Based Prognosis Framework for Energy Storage Devices With a Statistical Characterization of State-of-Health Regeneration Phenomena," IEEE Trans. Instrum. Meas., vol. 62, no. 2, Feb. 2013, pp. 364-376.

[2] S. J. Tong, A. Same, M. a. Kootstra, and J. W. Park, “Off-grid photovoltaic vehicle charge using second life lithium batteries: An experimental and numerical investigation,” Appl. Energy, vol. 104, Apr. 2013, pp. 740-750.

[3] L. Canals Casals, M. González Benítez, and B. Amante García, “A cost analysis of electric vehicel battery second life businesses," Lecture Notes in Management and Industrial Engineering, Project Management and Engineering Research 2014, 2016, pp. 129-141.

[4] C. Sen, S. Member, N. C. Kar, and S. Member, "Battery Pack Modeling for the Analysis of Battery Management System of a Hybrid Electric Vehicle," in Vehicle Power and Propulsion Conference (VPPC), 2009, pp. 207-212.

[5] J. De Hoog, K. Handberg, and R. Jegatheesan, "Demonstrating Demand Management : How Intelligent EV Charging Can Benefit Everyone,” in EVS27 Electric vehicle symposium, 2013, pp. 1-12.

[6] S. Ruthe, J. Schmutzler, C. Rehtanz, and C. Wietfeld, "Study on V2G Protocols against the Background of Demand Side Management,” Interoperability Bus. Inf. Syst., vol. 1, no. 1, 2011, pp. 33-44.

L. Canals Casals and B. Amante García, “A review of the complexities of applying second life electric car batteries on energy businesses," in Energy Systems Conference, 2014.

H. Cruz Gibert, M. Cruz Zambrano, L. Canals Casals, S. Castella Daga, and P. Diaz Pinos, "Sunbatt: Use of a Second Life Battery System from PHEV in Stationary Applications," in Smart City Expo World Congress, 2015.

Y. Zhu, F. Zhuo, and L. Xiong, "Communication Platform for Energy Management System in a Master-slave Control Structure Microgrid," in 7th International Power Electronics and Motion Control Conference - ECCE Asia, 2012, pp. 141-145.

[10] L. Canals Casals, B. Amante García, S. Castellà Dagà, and M. Cruzzambrano, "Energy businesses from re-used electric vehicle batteries," in Global Cleaner Production and Sustainable Consumption Conference, 2015.

[11] A. Jossen, "Fundamentals of battery dynamics," J. Power Sources, vol. 154, no. 2, pp. 530-538, 2006.

A. Barré, B. Deguilhem, S. Grolleau, M. Gérard, F. Suard, and D. Riu, "A review on lithium-ion battery ageing mechanisms and estimations for automotive applications," J. Power Sources, vol. 241, 2013, pp. 680-689.

[13] J. Remmlinger, M. Buchholz, M. Meiler, P. Bernreuter, and K. Dietmayer, "State-of-health monitoring of lithium-ion batteries in electric vehicles by on-board internal resistance estimation," $J$. Power Sources, vol. 196, no. 12, Jun. 2011, pp. 5357-5363..

[14] I. Kim, "A Technique for Estimating the State of Health of Lithium Batteries Through a Dual-Sliding-Mode Observer," IEEE Trans. Power Electron., vol. 25, no. 4, Apr. 2010, pp. 1013-1022.

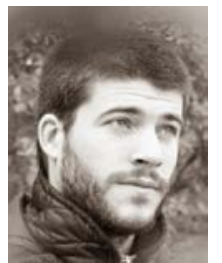

Lluc Canals Casals was born in Barcelona. He finished his master degree in industrial engineering in 2005 at the Universitat Politècnica de Catalunya and, after some years working in the automotive industry, in 2016 he became Doctor on electric vehicle battery ageing by the UPC.

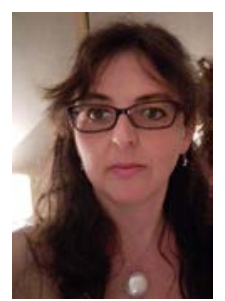

Beatriz Amante García was born in Alicante (Spain). She has a $\mathrm{PhD}$ in Telecommunications by the "École nationale supérieure des telecommunications" of Paris. She is nowadays working at the "Universitat Politècnica de Catalunya" in the Projects Engineering Department. She started her teaching career focusing on the fields of electronics, radio systems and Engineering projects. During these last years she started the study of battery ageing.
A. C. Karl VESTIN, "Networked Battery Management System, A novel and efficioent approach to Management of large battery systems," in Advanced Workshop: New challenges in Li-batteries materials and components for EVs (SOMABAT), Dec. 2013. 


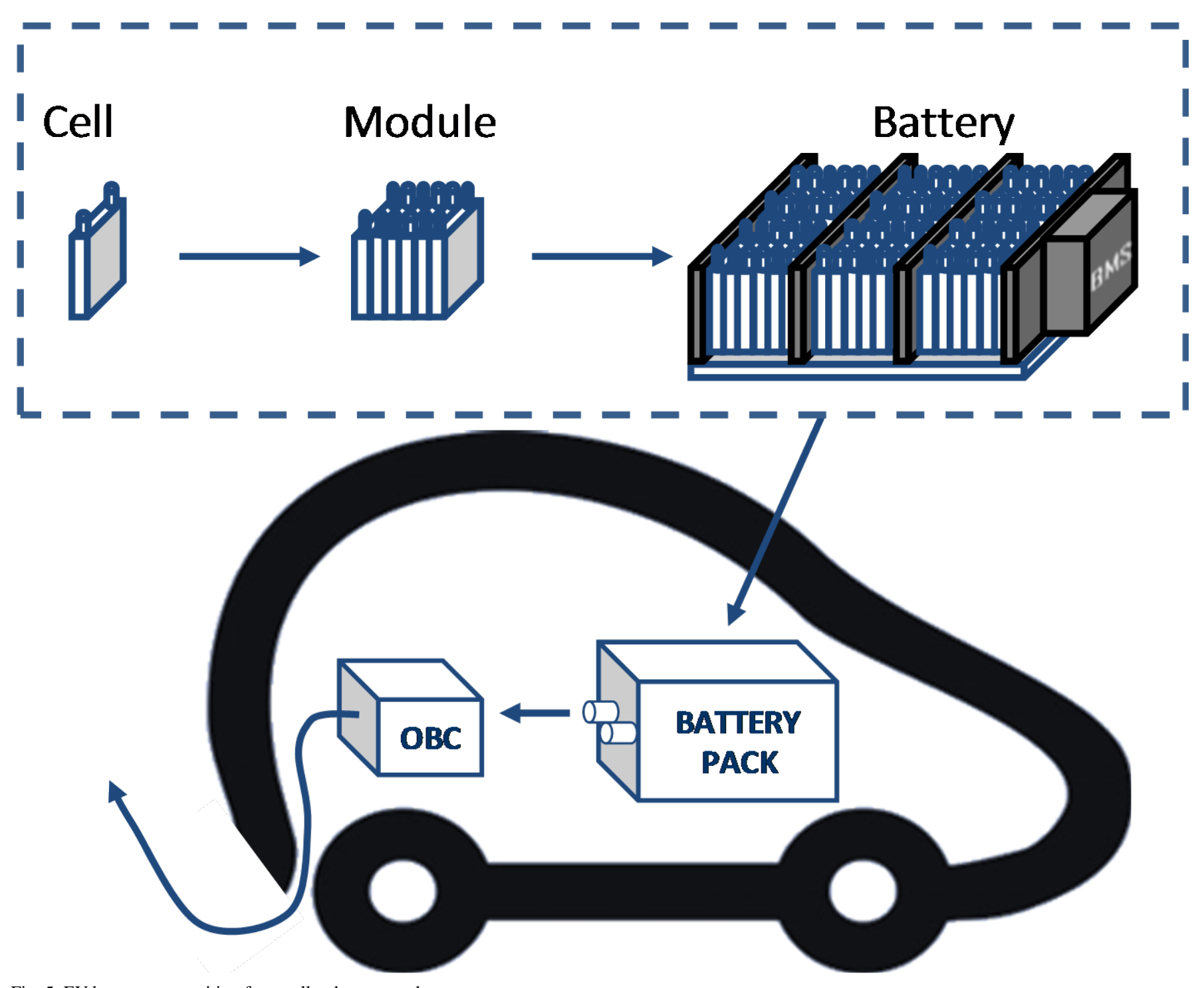

Fig. 5: EV battery composition from cell to battery pack. 
a)

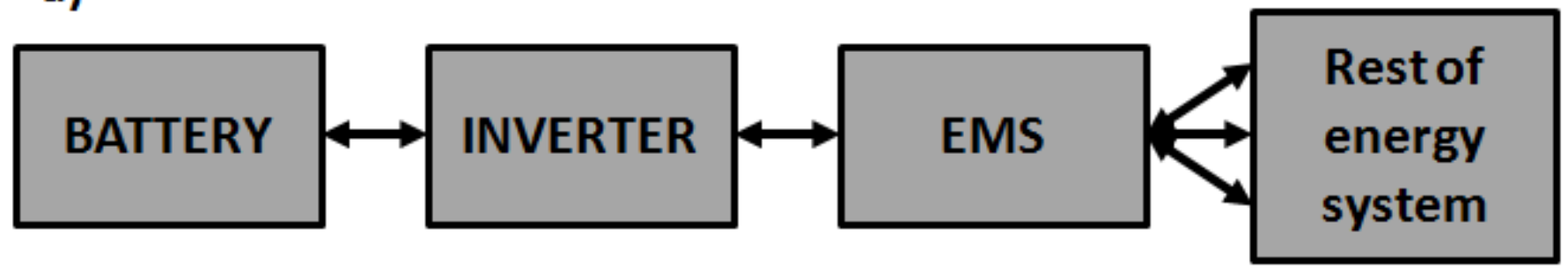

b)

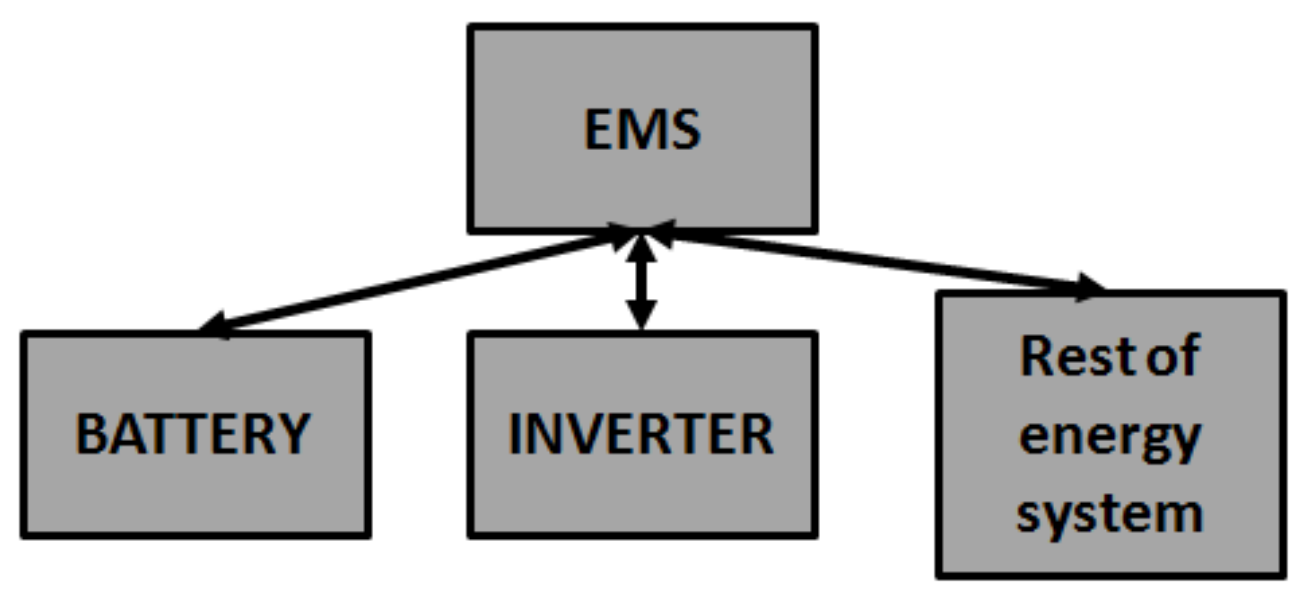

Fig. 6: Communications diagrams of the battery and the rest of elements in a grid. 

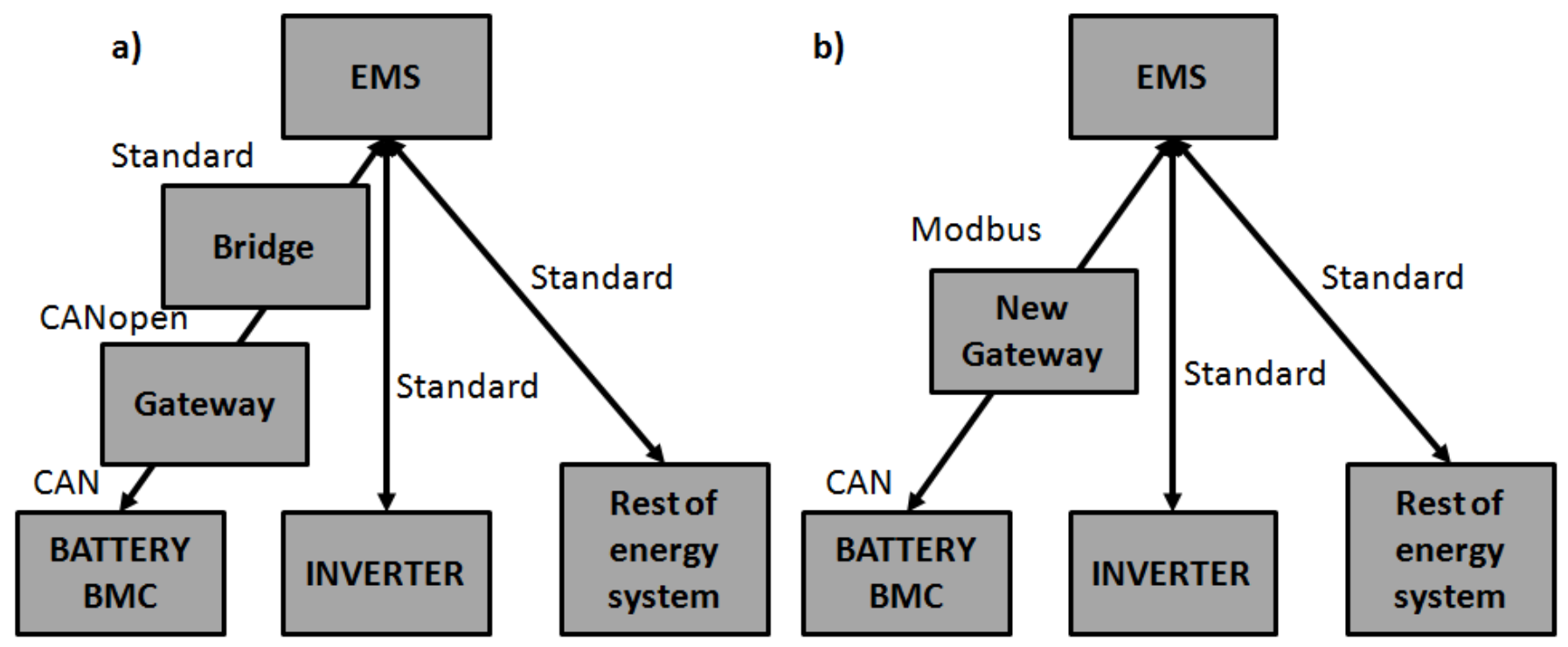

Fig. 7: Schema of the elements and the protocol used connected to the EMS. 


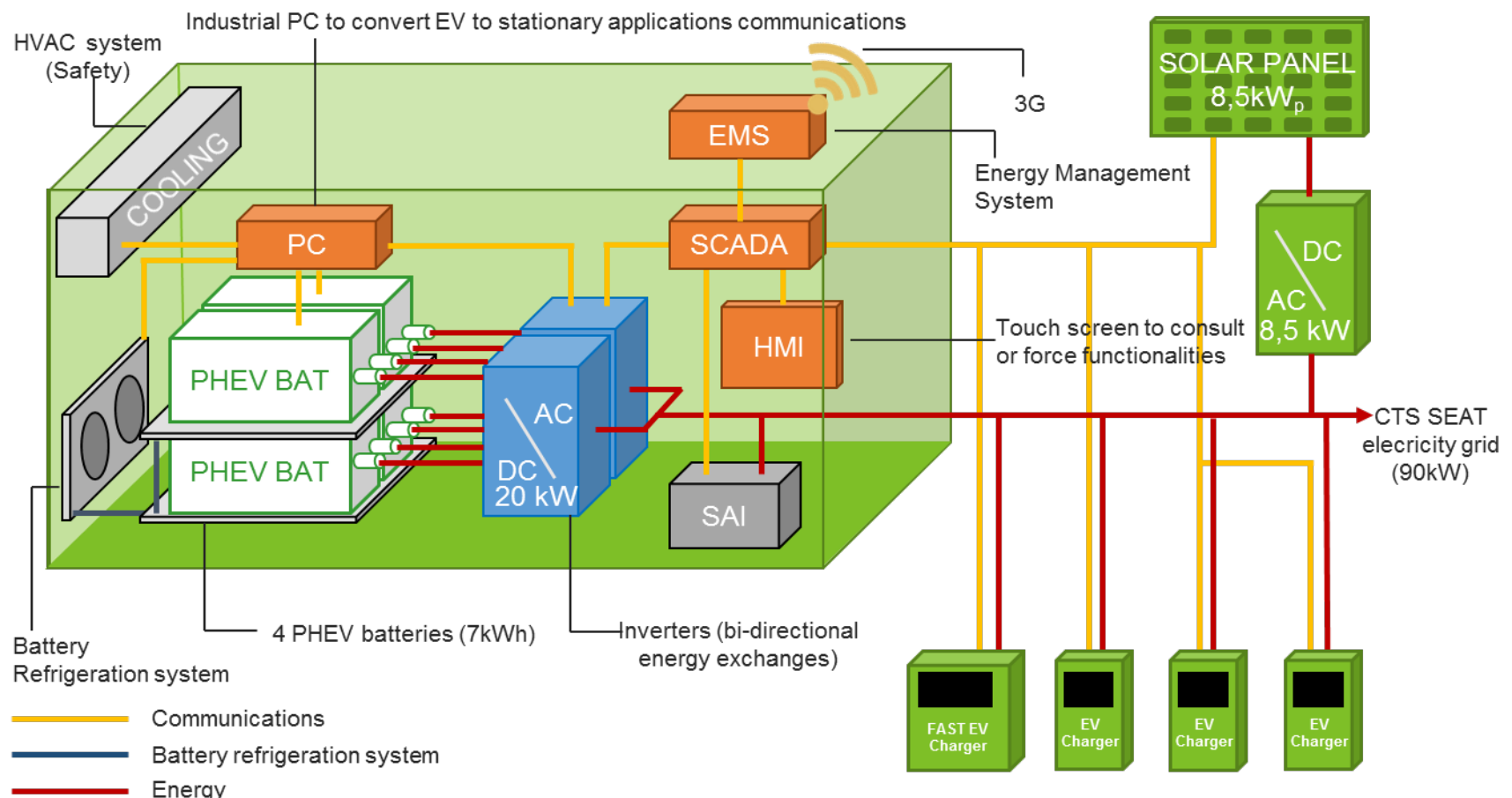

Fig. 8: Schema of the Sunbatt container connected to 3 normal EV chargers, one Fast EV charger, solar panels and a local grid. 